\title{
Markterschließung in China für Klein- und mittlere Unternehmen des Landes Brandenburg (ausgewählte Beispiele)
}

\author{
Dipl.-Ökon. Helga Schwab
}

Im Rahmen des Projektes „Markterschließung in Ost- und Südostasien für kleinere und mittlere Unternehmen KMU in betrieblicher Kooperation und Erprobung praxisnaher Methoden der Fachhochschulbildung" erfolgten seitens des Institutes für Internationales Marketing und Unternehmensführung an der Technischen Fachhochschule Wildau konkrete Aktivitäten, entsprechende Firmen des Landes Brandenburg nach China zu fuihren.

Zum einen lag das Interesse darin begründet, kleinen und mittelständischen Firmen die Möglichkeit zu erschließen, ihren bisherigen Export auf den asiatischen Markt auszudehnen, und zum anderen darin, als Markt das Land China auszuwählen, das Land Asien, welches gegenwärtig gute Aussichten auf einen erfolgreichen Markteinstieg bietet. Worin liegen diese guten Aussichten begründet?

- China ist ein sehr großes Land, nach Rußland und Kanada drittgrößtes Land der Welt. Es umfaßt eine Fläche von 9,6 Mio. $\mathrm{km}^{2}$, das sind $6,5 \%$ der Landoberfläche der Erde. $98 \%$ der Fläche befindet sich zwischen dem 20. und 50. nördlichen Breitengrad, es hat eine Ausdehnung von West nach Ost von 5.200 $\mathrm{km}$ und von Nord nach Süd von $5.500 \mathrm{~km}$. Seine Festlandsgrenzen betragen $22.000 \mathrm{~km}$ und seine Küstengrenzen $18.000 \mathrm{~km}$.

Die Regierungsadministration verläuft auf drei Ebenen: Provinzen (sowie Autonome Regionen und Städte, welche der Regierung direkt unterstellt sind), Kreise und Städte. Es sind 23 Provinzen, 6 Autonome Regionen und 4 der Regierung direkt unterstellte Städte.

1996 verzeichnete China 1,3 Mrd. Einwohner, für das Jahr 2010 werden 1,6 Mrd. geschätzt.

- China verzeichnet seit dem Beginn seiner Politik der Öffnung nach außen und der Durchführung von Reformen seit dem Jahre 1978 kontinuierlich ein zunehmend starkes wirtschaftliches Wachstum. Allein die Wachstumsraten 1996 im Bruttosozialprodukt betrugen $9,7 \%$, in der industriellen Produktion $13 \%$. Positionen wie zum Beispiel

Stahl. 100 Mio. $\mathrm{t}$

Walzstahl 85 Mio. $t$ chem. Düngemittel 28 Mio. $t$ Zement 471 Mio. $t$ wurden in großem Umfang produziert.

Ausgehend von diesen Grundgedanken ging es darum, Ansatzpunkte für ausgewählte brandenburgische Unternehmen zu finden, welche mit der gegenwärtigen Interessenlage in China korrespondieren. Drei Beispiele sollen nachfolgend dargestellt werden.

\section{Firma UGS Untergrundspeicher- und Geo- technologiesysteme GmbH Mittenwalde}

Der Hauptanteil der Produktion dieser Firma liegt im Bau von unterirdischen Speichern für die Lagerung von Erdgas. Es galt deshalb, Arbeitsbeziehungen im Bereich des Energiesektors insbesondere auf dem Gebiet der Erdgasproduktion herzustellen.

Zur Marktsituation wurde wie folgt recherchiert: Als die Volksrepublik China im Jahre 1949 gegründet wurde, war die Energieindustrie Chinas äußerst gering entwickelt.

Primärenergieproduktion 23,74 Mtce

Produktion von Rohkohle

Rohöl $0,12 \mathrm{Mt}$

Erdgas $7 \mathrm{Mm}^{3}$

Hydroenergie

0,7 Twh

In den Jahren 1949 bis 1995 hat Chinas Energiesektor eine schnelle Entwicklung genommen: Primärenergieproduktion 1048,4 Mtce. Die jährliche Wachstumsrate zwischen 1949 und 1991 betrug ca. $9 \%$. Der Anteil an der Primärenergieproduktion betrug 1991 aus

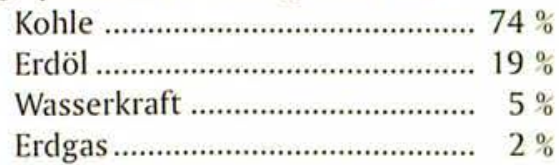

Von 1949 bis 1991 stieg Chinas Produktion an Rohkohle von $32 \mathrm{Mt}$ auf $1087,4 \mathrm{Mt}$ $\begin{array}{ll} & \text { (auf den 1. Platz in der Welt) } \\ \text { Erdöl } & \text { von } 0,12 \mathrm{Mt} \text { auf 140,9 Mt }\end{array}$ (auf den 5. Platz in der Welt)

Erdgas $\quad$ von $7 \mathrm{Mrd} . \mathrm{m}^{3}$ auf ca. $15 \mathrm{Mrd} . \mathrm{m}^{3}$ (Platz 20 in der Welt)

Weitere Entwicklung

\begin{tabular}{lccc}
\multicolumn{4}{c}{ Produktion an } \\
& Kohle (Mt) & Erdöl (Mt) & Erdgas (Mrd. $\mathrm{m}^{3}$ ) \\
\hline 1992 & 1114,5 & 141,1 & 15,7 \\
1993 & 1149,7 & 145,2 & 16,3 \\
1994 & 1229,5 & 146,1 & 16,7 \\
1995 & 1300,0 & & \\
& (USA 800 Mt) & 148,8 & 17,0 \\
1996 & 1380,0 & 155,0 & 20,1
\end{tabular}

Der Energieverbrauch in China unterscheidet sich von den Industrieländern und anderen Entwicklungsländern besonders darin, daß

- vorwiegend landeseigene Energiereserven genutzt werden, 
- Kohle die Primärenergieversorgung darstellt,

- ein extrem hoher Verbrauch an Bioenergie existiert,

- die Pro-Kopf-Produktion an Energie weit unter dem Weltdurchschnitt liegt,

- der Energieverbrauch Pro-Kopf-Bruttosozialprodukt höher ist als in entwickelten Ländern.

Im wesentlichen wird China seinen steigenden Bedarf an Energie weiterhin mit Kohle decken. Die Mengen dafür sind vorhanden. Die wirtschaftlich gewinnbaren Vorräte werden auf $62 \mathrm{Mrd}$. $\mathrm{t}$ geschätzt, das sind $12 \%$ der Weltreserven.

Das Problem in China besteht jedoch darin, daß die Hauptmasse der Kohle in anderen Gebieten liegt, als dort, wo sie verbraucht wird. In den Ostregionen Chinas liegt der Verbrauch, in den Nord- und Westregionen die Gewinnung.

Die großen Lagerstätten sind in der Provinz Shanxi (27\% der nationalen Förderung) und den Nachbarprovinzen konzentriert. Aus dieser Region muß die Kohle bis zu den Industriezentren per Bahn und Schiff im Schnitt $2.000 \mathrm{~km}$ transportiert werden. Chinas Eisenbahnnetz ist $\mathrm{zu} 40 \%$ mit dem Transport von Kohle ausgelastet.

Das Energieproblem ist einer der entscheidenden Punkte für die Modernisierung Chinas. Chinas Industrialisierung ist eingetreten in eine Periode von der arbeitsintensiven zur energieintensiven. Eine energieintensive Industrie und ein entsprechender Transport brauchen eine stabile Basis, wenn hohe Wachstumsraten erreicht werden sollen. Es wird eingeschätzt, daß der Energiebedarf Chinas im Jahre 20001.400 bis 1.700 Mtce betragen wird, das mögliche Eigenaufkommen könnte bei 1.400 Mtce liegen.

Infolge der raschen wirtschaftlichen Entwicklung unternimmt China große Anstrengungen, weitere Energiequellen auszubauen (Erdöl, Hydroenergie, Erdgas und Kohlegas). Die Nutzung von Erdgas wurde in den vergangenen Jahrzehnten in China wenig beachtet. Es wurden so gut wie keine Investitionen in die Produktion von Erdgas vorgenommen. Kleine Erdgasvorkommen wurden in China bisher nur im Süden des Landes entdeckt, wo man anfangs größere Vorkommen vermutete. Gegenwärtig unternimmt China jedoch große Anstrengungen zur Erkundung weiterer Erdgasvorkommen. Es wird eingeschätzt, daß sich in der Provinz Xinjiang (Westchina) und in der Provinz Innere Mongolei einige größere Erdgaslagerstätten befinden. Um das in diesen Gebieten befindliche Erdgas nach dem Abbau in die Industriezentren des Ostens zu bringen, plant man bereits den Bau einer Erdgasleitung von Westchina nach Ostchina mit einer Länge von $5.000 \mathrm{~km}$. Desweiteren plant man den Bau einer Erdgasleitung aus Zentralchina, entsprechende Vorkommen entdeckte man 1996, nach Peking und Tianjin mit einer Länge von $1.000 \mathrm{~km}$. Für diese Städte entwickelte man neue umweltfreundliche Energiesysteme.

Um einen schnellen Durchbruch bei der Nutzung von Erdgas zur teilweisen Sicherung des Energiebedarfs zu erreichen, orientiert sich die chinesische Regierung gleichzeitig auf den Import von Erdgas. So erfolgte Ende
1996 der Abschluß einer Vereinbarung zwischen Rußland und China über den Bau einer Erdöl- und Erdgasleitung sowie einer Leitung fuir Strom. Diese Erdöl- und Erdgasleitung, Länge 6.000 km, wird von Rußland, Ostsibirien, über die Mongolei bis zur chinesischen Pazifikküste verlaufen. Die Inbetriebnahme der Erdölleitung soll im Jahre 2005 und die der Erdgasleitung im Jahre 2002 erfolgen. Anläßlich des Besuches des chinesischen Regierungschefs Jiang Zemin in Moskau im November 1997 erfolgte die Unterzeichnung dieses Vertrages.

Die Kontaktaufnahme des Institutes für Internationales Marketing mit den entsprechenden staatlichen Stellen in China und den zuständigen Firmen erfolgte Ende 1996. Alle aufgesuchten Gesprächspartner zeigten sich erfreut und interessiert am Auftreten des Institutes in China sowie an der beauftragten Untersuchung und Kontaktanbahnung für die Firma UGS Untergrundspeicher- und Geotechnologie-Systeme GmbH Mittenwalde. Man bezeichnete es als eine große Chance für die Firma UGS Mittenwalde, nach Peking zu kommen.

In den Gesprächen wurde informiert, daß es bisher keinen einzigen Untergrundspeicher für Gas in China gäbe. Technische Erfahrung dabei wäre sehr notwendig, da die Leitung nicht nur außerordentlich lang und die Temperaturunterschiede sehr groß wären, sondern auch die Höhenunterschiede eine Kompliziertheit für sich allein seien.

Die Firma UGS Mittenwalde wurde eingeladen, nach Peking zu kommen, um in mehrtägigen Gesprächen und einem Symposium mit Verantwortlichen und Fachleuten für den Bau von Erdgasleitungen das Leistungsangebot der Firma UGS Mittenwalde vorzustellen. Die chinesische Seite erklärte sich gleichzeitig bereit, ihrerseits entsprechende Projekte vorzustellen.

\section{Firma BE Maschinenmesser GmbH \& Co. KG}

Das Produkt dieser Firma sind Form- und Flachmesser für die Lebensmittel-, insbesondere die Fleischindustrie.

Die Recherchen zur Vorbereitung der Gespräche befaßten sich mit der Fleischproduktion und Fleischverarbeitung Chinas. Sie ergab folgendes Ergebnis: Chinas Fleischproduktion umfaßt den Viehbestand, die Viehschlachtung, die Fleischverarbeitung, die Produktion, die Produktion von Nebenprodukten, die Kühlung und das Einfrieren, die Kühlhauslagerung sowie die Verpakkung. In den vergangenen rund 20 Jahren ist die Fleischproduktion jählich kontinuierlich gestiegen, sie betrug
1978 9,5 Mio. t
1982 12,0 Mio. $t$
1985 19,3 Mio. t
1986 21,1 Mio. $t$
198926,3 Mio. $t$
199234,4 Mio. $t$
1994 43,0 Mio. $t$ (die USA überholend)
199660,0 Mio. $t$ (entspricht $1 / 3$ der Weltproduktion)

geplant $2000 \quad 68,0$ Mio. t 


$\begin{array}{lcr}\text { Steigerungsrate: } & 1989 / 1996 & 130 \% \\ & 1978 / 1996 & 530 \% \\ \text { jährlicher Pro-Kopf- } & & \\ \text { Fleischverbrauch: } & 1985 & 18,5 \mathrm{~kg} \\ & 1994 & 35,0 \mathrm{~kg} \\ & & \end{array}$

$10 \%$ der Deviseneinnahmen Chinas resultieren aus dem Export von Schweinen und Schweinefleisch. In China steht die Fleischindustrie an vorrangiger Stelle bei der Nutzung von Devisen für die Modernisierung dieses Industriezweiges.

In den 70er Jahren baute China eine Anzahl Schlachthöfe und Fleischverarbeitungsfabriken. In den $80 \mathrm{er}$ Jahren entwickelten sich in China, verbunden mit seiner rapiden ökonomischen Entwicklung, neue Technologien der Fleisch- und Geflügelaufbereitung sowie der Kühlhauslagerung im großen Maße. Bereits 1985 gab es 1.240 Fleischverarbeitungsfabriken in China. 1997 gibt es inzwischen 1.600 .

\begin{tabular}{lrr} 
Fleischverarbeitung pro Schicht: & 1985 & 1994 \\
\hline Schweine & 511.000 & 690.000 \\
Rinder & 16.000 & 20.000 \\
Schafe & 170.000 & 200.000 \\
Geflïgel & 800.000 & 1.000 .000 \\
Fleisch & & $900 \mathrm{t}$ \\
Fleischprodukte & & $1000 \mathrm{t}$
\end{tabular}

Gegenwärtig konzentriert sich die Entwicklung der Fleischverarbeitung auf die Zerlegung von Fleisch, die Fleischkühlung, das Einfrieren von Frischfleisch, die Produktion kleiner Fleischpackungen, alles für mittlere und große Städte. Der Mangel an gekochten Fleischprodukten und Büchsenfleisch ist groß.

In China beträgt der Anteil von industriell verarbeitetem Fleisch gemessen an der Gesamtproduktion weniger als $10 \%$ (in hochentwickelten Ländern beträgt dieser Anteil 40 bis $70 \%$ ).

Die hauptsächlichsten Fleischverarbeitungsausrüstungen, die in China produziert werden, sind: Fleischwöl$\mathrm{fe}$, Fleischschneidemaschinen, Fleischschneide- und -mischmaschinen, Würstchenmaschinen, Infrarot-Ofen, Räucherofen und Schlachtausrüstungen für Schweine, Rinder, Schafe und Geflügel, jedoch die meisten Ausrüstungen sind mittlere und kleine mit geringer Produktionskapazität und wenigen Funktionen. Große und moderne Maschinen und Ausrüstungen wurden meistens aus dem Ausland importiert. Mehr als 200 Firmen in 21 Provinzen und Städten importierten entsprechende Ausrüstungen, im Jahre 1991, 1992 und 1993 für 70 Mio. US-Dollar sowie Fleischverarbeitungsanlagen für 90 Mio. US-Dollar hauptsächlich aus Deutschland, Dänemark, den Niederlanden, Frankreich, Österreich, Italien, Schweiz, Japan und USA. Diese Maschinen waren: Salzinjektoren, Fleischmischmaschinen, Fleischschneideund -mischmaschinen, Vakuum-Fleischknetmaschinen, Fleischfüllmaschinen, Vakuum-Verpackungsmaschinen usw., mehr als 200 Stück, darunter 47 „Western-Style“Würstchenproduktionslinien, 30 „Western-Style“-Schinkenproduktionslinien, einige Knochenverarbeitungsmaschinen sowie Prüf- und Testmaschinen.
Diese importierten Maschinen und Anlagen beeinflußten eine allgemeine Veränderung der Struktur der Fleischproduktion in China. Der Umfang von „WesternStyle"-Fleischprodukten wächst schneller als der Umfang der traditionellen chinesischen Fleischprodukte. Besonders in den vergangenen Jahren wuchs insbesondere der Anteil von Würstchen- und Schinkenprodukten, hergestellt unter hohen Temperaturen und in Hochdruck-Sterilisation in unerwartet hohem Maße.

Die Ergebnisse in Chinas Fleischproduktion waren 1995 wie folgt:

Fleischproduktion gesamt 47,0 Mio. t

Steigerung 1994/1995 4,4\%

Pro-Kopf-Verbrauch $\quad 39,3 \mathrm{~kg}(198012,8 \mathrm{~kg})$

Schweinefleisch

Rindfleisch

Hammelfleisch

Geflügelfleisch

$70,0 \%$ (am Gesamtaufkommen)

$7,0 \%$ (am Gesamtaufkommen)

$4,0 \%$ (am Gesamtaufkommen)

$17,0 \%$ (am Gesamtaufkommen)

Die Kontaktaufnahme des Institutes für Internationales Marketing und Unternehmensführung mit den entsprechenden staatlichen Stellen in China sowie den zuständigen Firmen erfolgte im Herbst 1997. Das Interesse aller Gesprächspartner an dem Erzeugnis der Firma BE Maschinenmesser $\mathrm{GmbH}$ war außerordentlich groß. Dergleichen Qualitätsmesser werden in China nicht produziert, sondern mit den Maschinen importiert (teilweise bis zu einer Ersatzteilhaltung bis zu 10 Jahren). Die Qualität der in China produzierten Messer (Hauptproduzent in Shanghai) ist abhängig vom verwendeten Einsatzmaterial gering (max. Umdrehungszahl 2000/min., sie sind außerdem leicht brüchig). Die Firma BE Maschinenmesser $\mathrm{GmbH}$ produziert diese Messer bis zu $6000 \mathrm{U} / \mathrm{min}$. bei Fleischtemperaturen bis zu minus 30 Grad.

Aus der Reihe der Gesprächspartner konnte eine Institution gefunden werden, welche sich als Vertretung der Firma BE Maschinenmesser GmbH eignet. Diese Institution verfügt über den umfangreichsten und intensivsten Kontakt zu den Fleischproduzenten und Fleischmaschinenherstellern Chinas. Eine entsprechende Verhandlungsaufnahme dazu war unmittelbar gegeben.

\section{Firma ANATEC GmbH}

Analysatoren und Technologien zur Rohstoffkontrolle, insbesondere in der Kohleindustrie

Auch hier wurden Recherchen zu Angaben des Industriezweiges durchgefuihrt, in welchem die Erzeugnisse der Firma ANATEC GmbH Einsatz finden können, somit in der Kohleindustrie.

Dazu folgendes Ergebnis: China hat innerhalb von 40 Jahren eine umfassende Kohleindustrie aufgebaut. Kohle nimmt in China eine bedeutende Position im Bereich der ökonomischen und gesellschaftlichen Entwicklung ein. Kohle ist nicht nur der Hauptrohstoff für die Brennstoffund Elektroindustrie, Kohle ist auch ein wichtiger Rohstoff für die chemische Industrie, Energielieferant für Haushalt und Export.

Nach wie vor ist Kohle der Hauptlieferant für Chinas 
Primärenergieproduktion. $76 \%$ des Verbrauchs an Primärenergie basiert auf Kohle (1996), ungefähr $60 \%$ Rohmaterial für die chemische Industrie und $80 \%$ der Haushaltsenergie resultieren aus Kohle.

Die Kohlegebiete in China umfassen ein Gebiet von ungefähr 0,55 Mio. $\mathrm{km}^{2}$. Sie erreichen eine Tiefe bis $4.500 \mathrm{~m} .75 \%$ der Kohlereserven sind Steinkohle, $12 \%$ Anthrazitkohle und $13 \%$ Braunkohle.

Geographisch befindet sich der Hauptanteil der Kohle im Nordwesten Chinas. Die Kohlereserven befinden sich in drei Gebieten:

Nordwest-/Nordostchina

darunter in den Provinzen Shanxi

$84 \%$

Shaanxi

$16 \%$

Der im Tagebau abzubauende Teil an Kohle in China ist relativ klein, er beträgt maximal $7 \%$ der gesamtem Kohlereserven, von denen der Anteil an Braunkohle wiederum $70 \%$ beträgt. Diese Resserven befinden sich in den Provinzen Innere Mongolei, Shanxi, Xinjian und Yunnan.

Chinas Kohleproduktion kann abhängig vom Management in zwei Teile unterteilt werden: in staatlich betriebene Kohlebergwerke und in Kohlebergwerke der örtlichen Provinzregierungen. Die in staatlich betriebenen Kohlebergwerken produzierte Kohle wird auch umfassend von staatlichen Organen vertrieben, die der provinzeigenen Kohlebergwerke entsprechend von Provinzen, Präfekturen oder Kreisen, kollektiveigenen oder dorfeigenen Kohlebüros. Die Struktur der Produktionstechnologien ist entsprechend hochmechanisiert, teilmechanisiert oder handwerklich betrieben.

1991, als die Rohkohleproduktion 1.087,4 Mt betrug, entsprach der Anteil staatlich-kontrollierter Kohlebergwerke 480,6 Mt, d. h. 44,2\% der Gesamtproduktion, örtliche Bergwerke 18,7\% und städtische/dörfliche sowie privateigene $36,7 \%$.

Der Anteil produzierter gewaschener Kohle betrug 1991 $195,8 \mathrm{Mt}$, d. h. 18,1\% der Gesamtproduktion, reine Kohle $81,4 \mathrm{Mt}$, davon wurden 74,2 Mt ausschließlich im Bereich der Metallurgie verbraucht.

1991 existierten 17 große staatlich-gelenkte Kohlebergwerke in China, jedes produzierte über $10 \mathrm{Mt}$ jährlich.

Anfang der 90er Jahre betrug die durchschnittliche Produktionskapazität der staatlichen Kohlebergwerke 1.259 t/Schicht, 19941.590 t/Schicht. Der Mechanisierungsgrad der Untertagebergwerksproduktion betrug $68,6 \%$, davon vollmechanisiert 37,6\%. Der durchschnittliche Aschegehalt der Kohle aus staatlichen Kohlebergwerken betrug 19,27\%.

China ist der größte Kohleverbraucher der Welt. 1990 erreichte der einheimische Kohleverbrauch 1.055,2 Mt, davon 28,6\% für die Elektroenergieerzeugung, 10,1\% für den Haushaltsverbrauch und $40,6 \%$ für den Verbrauch in der Industrie.

Verursacht durch den hohen Kohleverbrauch in den Städten sowie die Nutzung von Bioenergie auf dem Land, existieren in China große Energieumweltprobleme. Ca. $30 \%$ der Kohle werden in China direkt ver- brannt, das verursacht eine enorme Luftverschmutzung. Ganze Gebiete im Südwesten und im Süden Chinas sind durch sauren Regen beeinträchtigt. Gegenwärtig produzieren die staatlichen Kohlebergwerke 76 Mt Kohleabfall, 1.440 Mt Wasser und $5.000 \times 10^{6} \mathrm{~m}^{3}$ Methangas jährlich.

Der überdurchschnittliche Verbrauch von Biomasse verursacht einen Rückgang der Vegetation, der Verbrauch von Stroh, welches nicht dem Land zurückgeführt wird, verursacht Bodenzerstörung und eine Reduzierung organischer Bestandteile des Agrarlandes. Gegenwärtig betragen die nationalen Gebiete, in welchen der Boden zerstört ist, 150 Mha, der nationale Durchschnitt organischer Bestandteile des Agrarlandes liegt unter 1,5\%.

Die Lösung von Umweltproblemen, welche durch Energie verursacht werden, ist eine schwierige und langzeitige Aufgabe in China. Deshalb sind dringend die Entwicklung von Energie mit der Umwelt und der Ökonomie zu koordinieren. Darin eingeschlossen ist gleichermaßen die Energiestruktur sowie die ökonomische und technische Kooperation mit dem Ausland.

In den vergangenen Jahren hat China zunehmend großes Augenmerk auf die ökonomische und technische Kooperation insbesondere mit hochentwickelten Ländern gelegt, besonders beim Kauf technischer Projekte, der Lieferung von Ausrïstungen und der Bereitstellung von Technologien sowie dem Austausch von Fachkräften und Spezialisten. Der Energiesektor Chinas ist stark engagiert in der technischen Kooperation und unterhält viele Beziehungen zu staatlichen Organisationen anderer Länder, akademischen Institutionen, Firmen und internationalen Organisationen mit einem weitreichenden Spektrum des Austauschs von Besuchen und Informationen.

Für die Firma ANATEC GmbH wurden in Peking eine Reihe von Gesprächen geführt - sowohl auf der Ebene des Ministeriums für Kohleindustrie als auch bei Firmen und Institutionen. Auch hier zeigten sich alle Gesprächspartner sehr interessiert an der beauftragten Untersuchung und Kontaktanbahnung für die Firma ANATEC $\mathrm{GmbH}$.

In den Gesprächen wurde eingeschätzt, daß der Markt für Kohlemeßgeräte analog dem Produkt der Firma ANATEC GmbH in China ziemlich groß sei, vor allem mit der Zunahme marktwirtschaftlicher Erfordernisse. Abnehmer wären sowohl die Kohleproduzenten als auch Kraftwerke, welche in China bis zu $90 \%$ mit Kohle arbeiten wie auch Stahlwerke, Zementfabriken, Düngemittelfabriken usw., überall dort, wo Kohle im Einsatz sei. Sowohl vom Ministerium für Kohleindustrie als auch einem Forschungsinstitut für Kraftwerksanlagenbau erhielt die Firma ANATEC GmbH Einladungen zu einem Besuch in Peking, um vor ausgewählten Interessenten des Fachgebietes und Verantwortlichen eine technische Vorstellung/Symposium durchzuführen.

Entsprechende Gespräche über eine weitere Zusammenarbeit sind anschließend gegeben.

Zusammenfassend kann festgestellt werden, daß für alle drei der genannten Firmen die Kontakte zur Aufnahme konkreter Arbeitsbeziehungen hergestellt wurden. Un- 
terstiitzend für das Ergebnis war, daß die angebotenen deutschen technischen Erzeugnisse der genannten Firmen auf ein echtes Interesse der chinesischen Partner stießen, der chinesische Markt für die Erzeugnisse allgemein und im besonderen zum gegenwärtigen Zeitpunkt einen entsprechenden Bedarf hat und daß die Verfasserin dieses Artikels, welche die Verhandlungen durchführte, langjährig in China für Firmen des deutschen Maschinenbaus gearbeitet hat.

Daß in Vorbereitung der Gespräche für alle drei Firmen Unterlagen in chinesischer Sprache über das jeweilige Firmenprofil, die technische Beschreibung der Erzeugnisse und teilweise Preislisten oder Musterangebote erarbeitet und den chinesischen Partnern vorher uibergeben wurden, ist als unverzichtbare Voraussetzung anzusehen.

Mit den weiteren Gesprächen dieser Firmen in China beginnt die eigentliche konkrete kommerzielle Arbeit, deren Zeitdauer bis zum Erfolg unterschiedlich lang sein kann. Jedoch haben technische Erzeugnisse aus Deutschland wie auch die Zuverlässigkeit in der Zusammenarbeit mit deutschen Firmen einen sehr guten Ruf. Das sollte ermutigend für deutsche Firmen sein, auf den chinesischen Markt zu gehen.

\section{Verfasserin}

\section{Dipl.-Ökon. Helga Schwab}

Technische Fachhochschule Wildau Institut für Internationales Marketing und Unternehmensführung Fachbereich Wirtschaft, Verwaltung, Recht Bahnhofstraße 15745 Wildau

Tel. +49 (0) 3375 - 508325

Fax $+49(0) 3375-508388$ 